\title{
Der Einfluss von Nachtdiensten auf das Schlafverhalten
}

Dunn LK et al. Anesthesiology Resident Night Float Duty Alters Sleep Patterns. Anesthesiology 2019; 131: 401-409. doi: 10.1097/ ALN.0000000000002806

Die Arbeitszeitreformen in jüngerer Vergangenheit führten in vielen Ländern zu der Abschaffung von 24Stunden-Diensten und hin zu kürzeren Schichten. Besonders in Arbeitsbereichen mit hoher Arbeitsbelastung, z. B. der Intensivmedizin, gibt es heutzutage zumeist Mehrschichtmodelle. Durch diese Umstellung arbeiten die Mitarbeiter zumeist mehrere Tage am Stück, auch im Nachtdienst. Welche Auswirkung diese Arbeitszeitmodelle auf den Schlafrhythmus haben, wurde in der vorliegenden Studie von Dunn und Kollegen an der Universität von Virginia untersucht.

Für ihre Untersuchung rekrutierten sie 30 Assistenzärzte der Anästhesie (24 m, $6 w)$, wovon 29 die Studie komplett abschlossen. Das Durchschnittsalter lag bei $30 \pm 3$ Jahre. Insgesamt wurde das Schlafverhalten an 12 Tagen mit Hilfe des ZMachine Insight (General Sleep Corporation, USA), einem Ein-Kanal-EEG, gemessen. An Tag 1-3 fand die Basismessung statt, an Tag 4-9 die Aufzeichnung während der Nachtdienste und an Tag 10-12 die Erhebung in der Erholungsphase. Zusätzlich zu der EEG-Messung füllten die Teilnehmer noch die Ep-

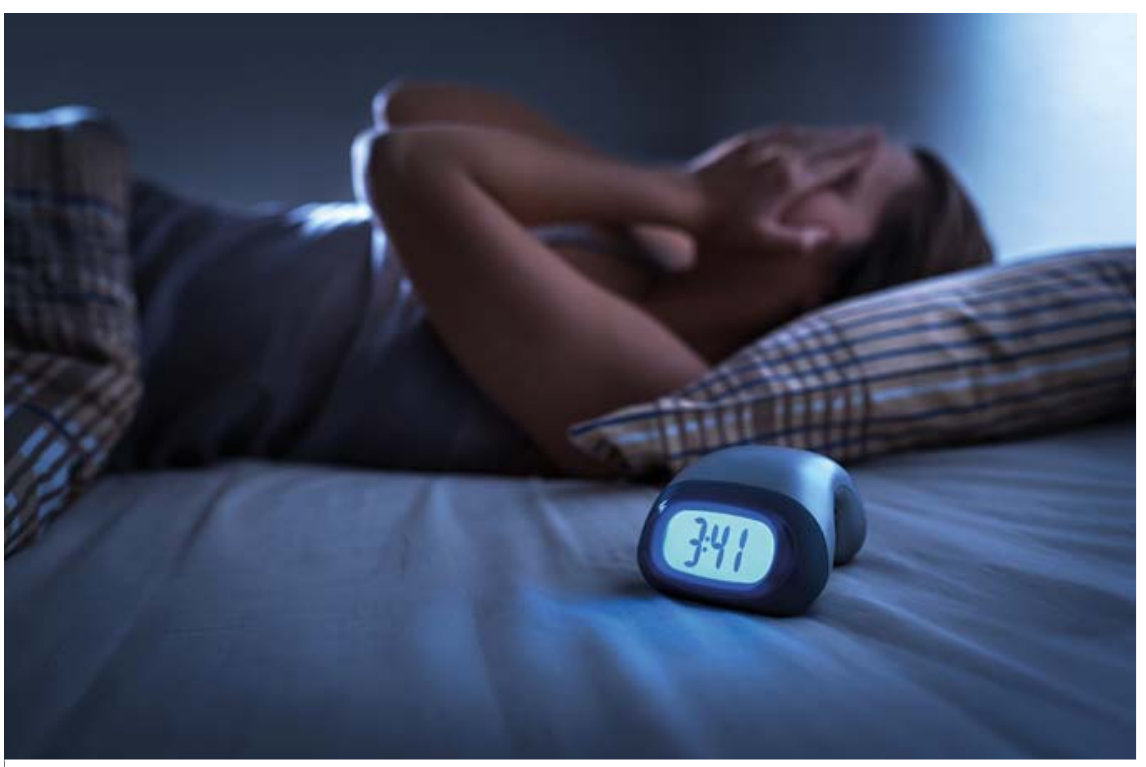

- Quelle: terovesalainen/stock.adobe.com worth Sleepiness Scale täglich aus, ein Messinstrument für die Erfassung der Tagesmüdigkeit (Gesamtpunktzahl von 0 24).

Die mittlere Schlafenszeit während der Basismessung betrug 5,9 1,9 Stunden, während der Nachtdienste sank diese um $1,4 \mathrm{~h}$ auf $4,5 \pm 1,8$ Stunden ab $(95 \%$ $\mathrm{KI}: 0,9-1,9 ; d=-1,1 ; \mathrm{p}<0,001)$. Besonders die Verringerungen in der Leichtschlafphase von 3,0 $\pm 1,2 \mathrm{~h}$ auf $2,2 \pm 1,1 \mathrm{~h}$ (ein Abfall um 0,7 h; 95\% Kl: 0,4-1,1; $d=-1,0$ ) und der REM-Phase (rapid eye movement) von $1,6 \pm 0,7 \mathrm{~h}$ auf $1,2 \pm 0,6 \mathrm{~h}$ (Abfall um 0,4 h; $95 \% \mathrm{Kl}$ : 0,3-0,6; $d=-0,9)$ waren nach der Bonferroni-Methode mit $p<0,001$ statistisch signifikant. Die Tiefschlafphase hingegen zeigte mit einem Abfall von 1,4 $\pm 0,6 \mathrm{~h}$ auf 1,1 $\pm 0,7 \mathrm{~h}$ (Abfall um 0,3 h; $95 \% \mathrm{Kl}$ : $0,1-0,4 ; d=-0,5 ; p<0,005)$ keine statistisch signifikante Änderung bei einem Bonferroni-Signifikanzniveau von 0,002.

In der Erholungsphase an Tag 10-12 war die mittlere Schlafenszeit mit 5,4 $\pm 2,2$ Stunden nicht signifikant anders als zur Basismessung vor den Nachtdiensten (Abfall um 0,5 h; 95\% Kl:-0,2-1,2; $d=-1,1 ; p=0,151)$. Es fiel allerdings ein statistisch signifikanter Abfall in der Tief- schlaf- und REM-Phase auf. Gerade diese beiden Phasen sind im Schlafzyklus für die Regeneration notwendig und ein Defizit kann auf eine beeinträchtigte Erholung hinweisen.

Auch in der Epworth Skala fielen statistisch signifikante Unterschiede in der Tagesmüdigkeit über den Erhebungszeitraum auf. Während der Basismessung betrug der durchschnittliche Wert 6,8 \pm 4,0 und stieg dann auf 10,0 \pm 4,9 (Zunahme von 3,2; $95 \% \mathrm{Kl}$ : 1,9-4,5; $d=0,9$; $\mathrm{p}<$ 0,001) während der Nachtdienstphase an. In der Erholungsphase fiel der Wert dann auf $8,7 \pm 4,6$ ab (Zunahme zur Basismessung von 2,0; $95 \% \mathrm{KI}:-3,5-0,5$; $d=0,9 ; p=0,02$ ).

Diese Untersuchung zeigt einige Besonderheiten auf. Schon während der Basismessung vor der Nachtdienstphase schliefen die Assistenzärzte weniger als 7 Stunden pro Nacht. Diese Erholungszeit wird jedoch von Schlafmedizinern empfohlen und eine dauerhafte Unterschreitung ist mit gesundheitlichen Risiken vergesellschaftet. Zwar kommt es in der Erholungsphase nach den Nachtdiensten wieder zu einer Zunahme der Schlafzeit, es fehlt aber dabei an den regenerierenden Tiefschlaf- und REM-Phasen.

\section{FAZIT}

Die Autoren schließen aus den Ergebnissen ihrer Studie sowie im Vergleich mit anderen Untersuchungen, dass eine Regenerationsphase von 3 Tagen nach einer Nachtdienstperiode nicht ausreichend ist, um einen gesunden Schlafrhythmus wiederherzustellen. In Folgestudien sollte somit das Augenmerk auf die optimale Erholungszeit sowie den Vergleich verschiedener Nachtschichtmodelle gelegt werden.

Dr. med. Christian Volberg, Marburg 


\section{Kommentar}

In der August-Ausgabe von Anesthesiology 2019 beschreiben die Autorengruppe um Lauren Dunn eine interessante Beobachtungsstudie zu Schlafveränderungen, verursacht über Nachtschichtdienstarbeiten bei Weiterbildungsärzten im 2. -4.Jahr der Weiterbildung an der University of Virginia, Charlottesville, Virginia USA. Dabei untersuchten die Autoren 30 Ärzte mit einem geplanten Einsatz von sechs Nächten in Folge im Schichtdienst (night float). Die Erhebungen zur Schlafqualität und Quantität wurden sowohl über ein EEG-prozessiertes Monitoring als auch über die sog. Epworth Sleepiness Scale erfasst. Individualisierte Normalbefundwerte wurden über Datenerhebungen an drei Tagen vor dem Schichtdienst gesammelt, Messwerte an sechs Tagen der Nachtschicht als auch nachfolgend zu Rekompensationsmechanismen im Anschluss zur Nachtschicht über drei weitere Tage erhoben. Die Ergebnisse lassen zunächst aufhorchen: Nach sechs Tagen Nachtschicht zeigten sich signifikante Veränderungen in der Schlafqualität als auch in der Schlafdauer bei den untersuchten Schichtarbeitenden Weiterbildungsärzten. Bei diesen Nachtschichten war zudem eine nachfolgende dreitägige Erholungszeit zu kurz, um auf die individualisierten Basiswerte der Schlafqualität und- Quantität zurück zu kehren. Keine Hinweise zur kognitiven Leistungsfähigkeit wurden in der Beobachtungsstudie gegeben.

Negative Schlafveränderungen, ungenügende Erholungsphasen, nachhaltige Veränderungen der zirkadianen Rhythmik sind stark mit Gesundheitsgefährdungen für den Einzelnen - im Berufsfeld Medizin besonders auch für den Patienten - verbunden. Zu groß sind jedoch die Unterschiede zur Arbeitsumgebung, Arbeitszeitregelung, Dienstbelastungen des amerikanischen Weiterbildungssys tem [1], als dass wir die Ergebnisse der Beobachtungsstudie zu einer direkten Vergleichbarkeit oder gar für mögliche Konsequenzen für Ärzte in der Fachgebietsweiterbildung Anästhesiologie in Deutschland übernehmen könnten. Zum einen finden sich in Deutschland erfreu- licherweise weitaus restriktivere normative Regulierungen zur Arbeitszeit und Arbeitsbelastung. Daraus ergeben sich gravierende Unterschiede in der Arbeitszeit (regulär 80 Wochenstunden USA; 1 freier Tag pro 7 Tage Woche) und der Arbeitsbelastung von amerikanischen Weiterbildungsärzten zu deutschen Weiterbildungsärzten. Zum anderen ist die Studie mit einigen methodischen Mängeln versehen (zu kurze Evaluationsphase, vorbestehende Schlafdeprivation, keine Bestimmung der vorbestehenden $\mathrm{Ar}$ beitsbelastungen, keine psychosoziale Evaluation bzgl. der vorbestehenden Schlafmangelzuständen, keine Medikamentenanamnese etc.).

2018 hatten die Delegierten des 121. Deutschen Ärztetages angemahnt, dass Arbeitsbedingungen in allen Sektoren und ärztlichen Berufsfeldern weder die körperliche noch die seelische Gesundheit von Ärzten gefährden dürften und minimiert werden müssten [2]. Nachtschlafveränderungen und Schlafdeprivationen stellen bekannte Ursachen für viele Gesundheitsschäden wie ein Burnout-Syndrom und Depression dar. Beide Erkrankungen sind im medizinischen Umfeld mit hoher Prävalenz anzutreffen [3].

Die Beobachtung der amerikanischen Kollegen sollte deshalb zu weiteren strukturierten wissenschaftlichen Untersuchungen zu den Auswirkungen von Nachtarbeit und Nachtschichtlängen im Krankenhaus in Deutschland führen. Nicht vergessen werden darf, dass ca. 3,5 Millionen Menschen Schichtarbeit bei uns leisten. Unsere gesamte Tagund-Nacht-Lebenswelt auch außerhalb der Kliniken wird über Nachtschicht-Arbeitende mit aufrechterhalten. Sollten notwendige Verbesserungen der Gesundheit in Deutschland über veränderte Konditionen der Nachtarbeit erreichbar werden, wird dies meines Erachtens nur mit erheblichen volkswirtschaftlichen Belastungen einerseits und deutlich veränderten individuellen Lebensgewohnheiten anderseits, einhergehen.

\section{Interessenkonflikt}

Die Autorinnen/Autoren geben an, dass kein Interessenkonflikt besteht.

\section{Autorinnen/Autoren}

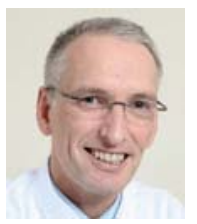

Univ.-Prof. Dr. med. Hartmut Bürkle, Klinik für Anästhesiologie und Intensivmedizin, Universitätsklinikum Freiburg

Literatur

[1] Desai $S$ et a. Education Outcomes in a DutyHour Flexibility Trial in Internal Medicine. N Engl J Med 2018; 378:1494-1508

[2] Richter-Kuhlmann E. Arztgesundheit: Selbstfürsorge kommt zu kurz. Dtsch Arztebl 2019; 116 (Sonderausgabe Arztgesundheit)

[3] Beschoner P, Limbrecht-Ecklundt K, Bretzke L]. Psychische Gesundheit von Ärzten. Der Nervenarzt 2019, 90: 961 - 974 